\title{
Dual role of the integrated stress response in medulloblastoma tumorigenesis
}

\author{
Sarrabeth Stone ${ }^{1,2,3, *}$, Yeung Ho ${ }^{1,2,3, *}$, Xiting Li $^{1,2,3,4}$, Stephanie Jamison ${ }^{1,2,3}$, Heather \\ P. Harding ${ }^{5}$, David Ron ${ }^{5}$, Wensheng Lin ${ }^{1,2,3}$ \\ ${ }^{1}$ Department of Neuroscience, University of Minnesota, Minneapolis, Minnesota, United States \\ ${ }^{2}$ Institute for Translational Neuroscience, University of Minnesota, Minneapolis, Minnesota, United States \\ ${ }^{3}$ Masonic Cancer Center, University of Minnesota, Minneapolis, Minnesota, United States \\ ${ }^{4}$ Department of Periodontics, Guanghua School of Stomatology, Hospital of Stomatology, Sun Yat-sen University, Guangzhou, \\ Guangdong, China \\ ${ }^{5}$ Cambridge Institute of Medical Research, University of Cambridge, Cambridge, United Kingdom \\ *These authors contributed equally to this work
}

Correspondence to: Wensheng Lin, email: linw@umn.edu

Keywords: medulloblastoma, integrated stress response, ER stress, GADD34, tumorigenesis

Received: May 27, $2016 \quad$ Accepted: September 01, $2016 \quad$ Published: September 06, 2016

\section{ABSTRACT}

In response to endoplasmic reticulum (ER) stress, activation of pancreatic ER kinase (PERK) coordinates an adaptive program known as the integrated stress response (ISR) by phosphorylating translation initiation factor $2 a$ (eIF2a). Phosphorylated eIF $2 a$ is quickly dephosphorylated by the protein phosphatase 1 and growth arrest and DNA damage 34 (GADD34) complex. Data indicate that the ISR can either promote or suppress tumor development. Our previous studies showed that the ISR is activated in medulloblastoma in both human patients and animal models, and that the decreased ISR via PERK heterozygous deficiency attenuates medulloblastoma formation in Patched1 heterozygous deficient (Ptch1+/-) mice by enhancing apoptosis of pre-malignant granule cell precursors (GCPs) during cell transformation. We showed here that GADD34 heterozygous mutation moderately enhanced the ISR and noticeably increased the incidence of medulloblastoma in adult Ptch1+/- mice. Surprisingly, GADD34 homozygous mutation strongly enhanced the ISR, but significantly decreased the incidence of medulloblastoma in adult Ptch1+/- mice. Intriguingly, GADD34 homozygous mutation significantly enhanced pre-malignant GCP apoptosis in cerebellar hyperplastic lesions and reduced the lesion numbers in young Ptch1+/mice. Nevertheless, neither GADD34 heterozygous mutation nor GADD34 homozygous mutation had a significant effect on medulloblastoma cells in adult Ptch1+/- mice. Collectively, these data imply the dual role of the ISR, promoting and inhibiting, in medulloblastoma tumorigenesis by regulating apoptosis of pre-malignant GCPs during the course of malignant transformation.

\section{INTRODUCTION}

Activation of pancreatic endoplasmic reticulum kinase (PERK), in response to endoplasmic reticulum (ER) stress, coordinates an adaptive program known as the integrated stress response (ISR, Figure 1) by phosphorylating the $\alpha$ subunit of the eukaryotic translation initiation factor 2 (eIF2 $\alpha)[1,2,3]$. Phosphorylation of eIF2 $\alpha$ inhibits global protein translation but stimulates the expression of certain stress-induced genes through induction of the transcription factor ATF4. On the other hand, phosphorylated eIF $2 \alpha$ (p-eIF $2 \alpha$ ) is quickly dephosphorylated by the protein phosphatase 1 (PP1) and growth arrest and DNA damage 34 (GADD34) complex [4, 5], allowing cells to recover from inhibition of global protein biosynthesis. Interestingly, induction of ATF 4 by p-eIF $2 \alpha$ stimulates the expression of the transcription factor CAATT enhancer binding protein homologous protein (CHOP), and results in upregulation of GADD34, which forms a tight autofeedback loop regulating activity of the ISR $[6,7,8]$. 
Several lines of evidence have suggested that the PERK-mediated ISR plays an important role in tumor growth and invasion, including regulating tumor cell viability, tumor cell invasion, and angiogenesis $[9,10,11]$. Interestingly, recent studies showed that the ISR is also involved in the process of malignant transformation by regulating cell apoptosis [12, 13, 14]. However, the effects of the ISR on tumor development are controversial $[15,16,17]$. Some reports showed that the ISR attenuates cell apoptosis during malignant transformation and/or after malignant transformation, resulting in enhanced tumor development $[10,12]$. In contrast, other reports showed that the ISR elevates cell apoptosis during malignant transformation and/or after malignant transformation, resulting in attenuated tumor development $[13,18]$.

Medulloblastoma can be classified into four major molecular subgroups based on the molecular profiling: sonic hedgehog (SHH) subgroup, WNT subgroup, subgroup 3, and subgroup 4 [19, 20]. Mice heterozygous for Patched1 (Ptch1+/-), a SHH receptor, are regarded as the best animal model to study the SHH subgroup of medulloblastoma [21, 22]. The development of medulloblastoma in Ptch $1+/-$ mice exhibits distinct steps of progression [23, 24, 25]. These mice display hyperplastic lesions of pre-malignant granule cell precursors (GCPs) in the external granular layer (EGL) of the cerebellum starting at the age of 3 weeks, and the lesions persist as late as 6 weeks of age. While a few hyperplastic lesions undergo cell transformation and progress to malignancy, the majority of the lesions regress over time.

Recent studies showed that the PERK-mediated ISR participates in medulloblastoma development [23, 26, 27]. Activation of PERK signaling has been observed in pre-malignant GCPs in young Ptch1+/- mice and in medulloblastoma cells in human patients and animal models [27]. Importantly, our previous study showed that PERK haploinsufficiency reduces the incidence of medulloblastoma in Ptch1+/- mice. Intriguingly, PERK haploinsufficiency enhances apoptosis of pre-malignant GCPs in young Ptch1+/- mice, but has no significant effect on medulloblastoma cells in adult mice [27]. In this study, we sought to better understand the effects of the ISR on the development of medulloblastoma in Ptch1+/- mice by exploiting GADD34 mutant mice. We found that GADD34 heterozygous mutation noticeably increased the incidence of medulloblastoma in Ptch1+/mice. Surprisingly, GADD34 homozygous mutation significantly decreased the incidence of medulloblastoma in Ptch1+/- mice, which was associated with enhanced apoptosis of pre-malignant GCPs in young mice. Additionally, GADD34 mutation, either heterozygous or homozygous, did not affect medulloblastoma cells in adult Ptch1+/- mice. Collectively, these data suggest a dual role of the ISR, promoting and inhibiting, in medulloblastoma tumorigenesis.

\section{RESULTS}

\section{Dual role of the ISR in medulloblastoma formation in Ptch1+/- mice}

Ptch1+/- mice develop symptomatic medulloblastoma typically between the ages of 2 and 8 months $[22,27,28]$. Our previous study demonstrated activation of the PERK-mediated ISR in medulloblastoma cells in adult Ptch $1+/-$ mice and in pre-malignant GCPs in young mice [27]. We also showed that GADD34 is upregulated in medulloblastoma in Ptch1+/- mice [27]. It is well documented that inhibition of GADD34, via either genetic or pharmacological approaches, elevates the ISR during ER stress [29, 30]. Gadd34 homozygous

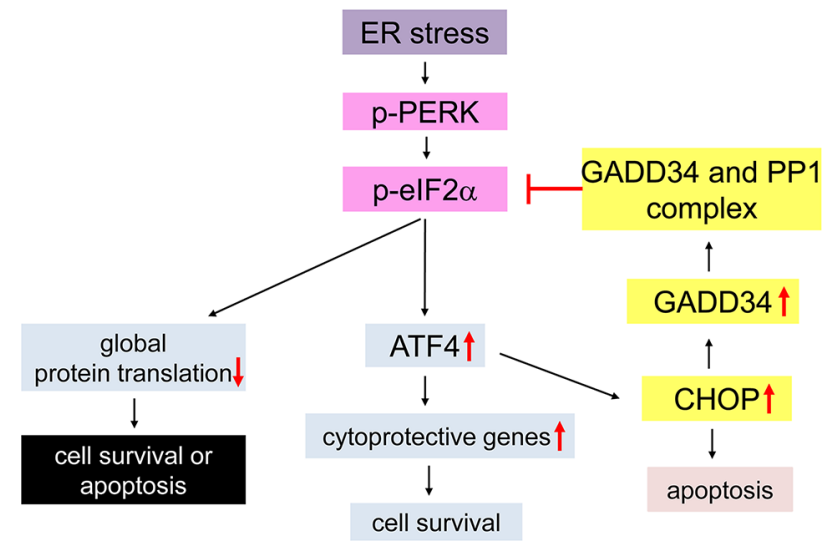

Figure 1: Schematic diagram of the PERK-mediated ISR. Under conditions of ER stress the PERK protein becomes activated by homodimerization and autophosphorylation (p-PERK). p-PERK phosphorylates eIF2 $\alpha$, and results in inhibition of global protein translation and induction of cytoprotective genes by preferentially stimulating translation of ATF4. ATF4 also enhances the expression of CHOP, which negatively regulates p-eIF $2 \alpha$ level through induction of GADD34 that binds PP1 and dephosphorylates p-eIF $2 \alpha$. The moderate ISR promotes cell survival by modestly inhibiting protein translation and enhancing the expression of certain cytoprotective genes. In contrast, the strong ISR leads to cell apoptosis by strongly inhibiting protein translation and/or inducing CHOP expression. 
mutant (Gadd34-/-) mice appear healthy and do not display any abnormalities in the CNS under normal conditions [7, 26, 29]. Moreover, a report showed that Gadd34 heterozygous mutant (Gadd34+/-) mice are healthy and display evidence of haploinsufficiency [31]. To determine the effects of the enhanced ISR on medulloblastoma development, Ptch $1+/-$ mice were crossed with Gadd34-/- mice, and then the resulting progeny were intercrossed to obtain Ptch1+/-; Gadd34 wild type $($ Gadd34+/+) mice, Ptch1+/-; Gadd34+/mice, and Ptch1+/-; Gadd34-/- mice. We monitored these mice daily up to 8 months to detect medulloblastoma phenotypes, including ataxia, decreased movement, poor grooming, and doming of the skull [21, 22]. We verified medulloblastoma formation in symptomatic mice by gross examination, namely determining enlargement of the skull and cerebellum, and by hematoxylin and eosin (H\&E) staining.

Our previous study showed that PERK heterozygous deficiency reduces the incidence of medulloblastoma in Ptch1+/- mice [27]. In agreement with this previous study, the frequency of symptomatic medulloblastoma in Ptch1+/-; Gadd34+/- mice was noticeably increased compared to Ptch1+/-; Gadd34+/+ mice (Figure 2A). We further performed necropsy on all 8-month-old asymptomatic mice. Gross examination and H\&E staining revealed that 2 out of 55 asymptomatic Ptch1+/-; Gadd34+/+ mice and 6 out of 45 asymptomatic Ptch1+/-; Gadd34+/- mice developed medulloblastoma, respectively. Thus, GADD34 heterozygous mutation noticeably increased the incidence of medulloblastoma in Ptch1+/- mice (29 out of 68 Ptch1+/-; GADD34+/mice vs 23 out of 76 Ptch1+/-; GADD34+/+ mice, Figure 2B). Western blot analysis showed that GADD34 heterozygous mutation did not change p-eIF $2 \alpha$ level, modestly elevated ATF4 level, and moderately increased CHOP level in medulloblastoma in Ptch1+/- mice (Figure 2C, 2D). It is known that elevation of p-eIF2 $\alpha$ level in ER-stressed cells is transient and highly dynamic; however, elevation of CHOP level is relatively steady and long-lasting [8, 32]. The moderate elevation of CHOP level indicates moderately enhanced ISR in medulloblastoma in Ptch1+/-; Gadd34+/- mice compared to Ptch1+/-; Gadd34+/+ mice. Taken together, these results suggest GADD34 heterozygous mutation results in the noticeable increase of medulloblastoma incidence in Ptch $1+/-$ mice by moderately enhancing the ISR.

Western blot analysis showed that GADD34 homozygous mutation did not alter the level of p-eIF2 $\alpha$ and moderately elevated the level of ATF4 in medulloblastoma in Ptch1+/- mice (Figure 2C). Importantly, the level of CHOP was strongly increased in medulloblastoma in Ptch1+/-; Gadd34-/- mice compared to Ptch1+/-; Gadd34+/+ mice (Figure 2C, 2D), suggesting that GADD34 homozygous mutation strongly elevates the ISR in medulloblastoma in Ptch1+/- mice. Surprisingly, the frequency of symptomatic medulloblastoma in Ptch1+/-; Gadd34-/- mice was significantly decreased compared to Ptch1+/-; Gadd34+/+ mice (Figure 2A). Moreover, gross examination and H\&E staining revealed that 4 out of 55 asymptomatic Ptch1+/-; Gadd34-/- mice developed medulloblastoma. Thus, GADD34 homozygous mutation significantly reduced the incidence of medulloblastoma in Ptch1+/- mice (9 out of 60 Ptch1+/-; Gadd34-/- mice vs 23 out of 76 Ptch1+/-; Gadd34+/+ mice, Figure 2B). Collectively, these results showed GADD34 homozygous mutation strongly enhances the ISR and results in the significant decrease of medulloblastoma incidence in Ptch1+/- mice.

\section{GADD34 mutation did not affect cell proliferation, cell apoptosis, or angiogenesis in medulloblastoma in adult Ptch1+/- mice}

Our previous study showed that the decreased ISR via PERK heterozygous deficiency has no effect on cell proliferation, cell apoptosis, or angiogenesis in medulloblastoma in adult Ptch1+/- mice [27]. We determined the effects of the enhanced ISR on medulloblastoma in adult Ptch $1+/-$ mice. H\&E staining showed that the morphology of medulloblastoma in both Ptch1+/-; Gadd34+/- mice and Ptch1+/-; Gadd34-/mice was comparable with Ptch1+/-; Gadd34+/+ mice (Figure 3A-3C). Immunohistochemistry (IHC) for glial fibrillary acidic protein (GFAP) and synaptophysin showed that the expression pattern of both GFAP and synaptophysin in medulloblastoma in both Ptch1+/-; Gadd34+/- mice and Ptch1+/-; Gadd34-/- mice was comparable with Ptch1+/-; Gadd34+/+ mice (Figure 3D-3I). Western blot analysis showed the protein levels of GFAP and synaptophysin were comparable in medulloblastoma in these three groups of mice (Figure 3J). Bromodeoxyuridine (BrdU) labeling (Figure 4A-4C, 4M) and proliferating cell nuclear antigen (PCNA) IHC (data not shown) showed that GADD34 mutation, either heterozygous or homozygous, did not significantly affect the number of proliferating cells in medulloblastoma in Ptch $1+/-$ mice. Moreover, terminal deoxynucleotidyl transferase-mediated biotinylated UTP nick end labeling (TUNEL) staining showed that GADD34 mutation, either heterozygous or homozygous, did not significantly alter cell apoptosis in medulloblastoma in Ptch1+/mice (Figure 4D-4F, 4M). Several studies showed that the ISR stimulates angiogenesis in tumors, including medulloblastoma, by enhancing the expression of vascular endothelial growth factor A (VEGF-A) [26, 33]. However, VEGF-A IHC showed that GADD34 mutation, either heterozygous or homozygous, had no significant effect on VEGF-A expression in medulloblastoma in Ptch1+/- mice (Figure 4G-4I, 4M), and CD31 IHC showed that GADD34 mutation, either heterozygous or homozygous, did not significantly affect angiogenesis in 
this tumor (Figure $4 \mathrm{~J}-4 \mathrm{~L}, 4 \mathrm{~N}$ ). Collectively, these data suggest that GADD34 mutation, either heterozygous or homozygous, has a minimal effect on cell proliferation, cell apoptosis, and angiogenesis in medulloblastoma in adult Ptch $1+/-$ mice. Thus, it is unlikely that GADD34 mutation influences medulloblastoma formation in Ptch1+/- mice through its effects on tumor cells.

\section{GADD34 homozygous mutation increased pre- malignant GCPs apoptosis in the cerebellum of young Ptch1 +/- mice}

Medulloblastoma arises from GCPs in the cerebellar EGL of Ptch1+/- mice and exhibits distinct steps of progression [23, 24, 25]. GCPs proliferate, differentiate, migrate to the internal granule layer, and become mature granule neurons during early postnatal development. There are no GCPs in the EGL of normal mice at the age of 3 weeks [34]. Nevertheless, Ptch1+/- mice display hyperplastic lesions of pre-malignant GCPs in the cerebellar EGL starting at the age of 3 weeks, and the lesions persist as late as 6 weeks of age [23, 25, 35]. The majority of these hyperplastic lesions regress over time; however, a few hyperplastic lesions undergo cell transformation and progress to malignancy [25, 36]. Our previous study showed that the decreased ISR via PERK heterozygous deficiency enhances pre-malignant GCP apoptosis, resulting in the reduction of medulloblastoma incidence in Ptch1+/- mice [27]. We further determined the actions of the enhanced ISR in pre-malignant GCPs in young Ptch1+/- mice.

We serially sectioned whole paraffin-embedded half-cerebellum of 6-week-old mice, each half-cerebellum yielded $\sim 2105$ - $\mu$ m-thick sections. Every tenth section in the series was either stained by H\&E or immunostained by the NeuN antibody or PCNA antibody. The hyperplastic
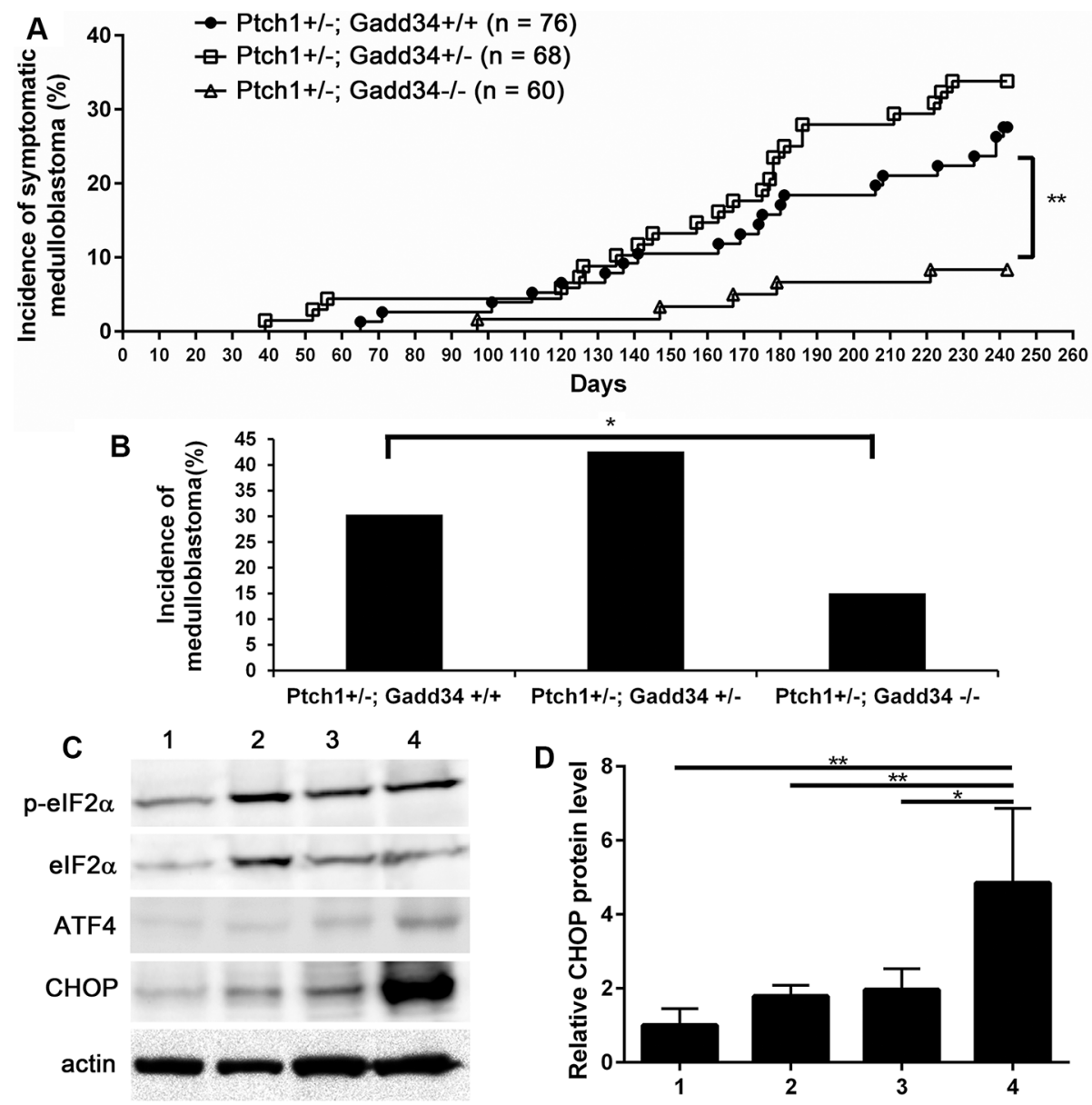

Figure 2: GADD34 mutation influenced medulloblastoma formation in Ptch1+/- mice. (A) The percentage of mice that developed symptomatic medulloblastoma by the age of 8 months. (B) The percentage of mice that developed medulloblastoma by the age of 8 months. Ptch1+/-; Gadd34+/+ mice, $n=76$ animals; Ptch1+/-; Gadd34+/- mice, $n=68$ animals; Ptch1+/-; Gadd34-/- mice, $n=60$ animal. (C, D) Western blot analysis showed that GADD34 heterozygous mutation did not change p-eIF2 $\alpha$ level, modestly elevated ATF4 level, and moderately increased CHOP level in the cerebellum of adult Ptch1+/- mice with medulloblastoma, and that GADD34 homozygous mutation did not change p-eIF2 $\alpha$ level, moderately elevated ATF4 level, and strongly increased CHOP level in the cerebellum of adult Ptch1+/- mice with medulloblastoma. 1, wild type mice; 2, Ptch1+/-; Gadd34+/+ mice; 3, Ptch1+/-; Gadd34+/- mice; 4, Ptch1+/-; Gadd34-/- mice. $N=4$ animals. Error bars represent $\mathrm{SD},{ }^{* *} P<0.01,{ }^{*} P<0.05$. 

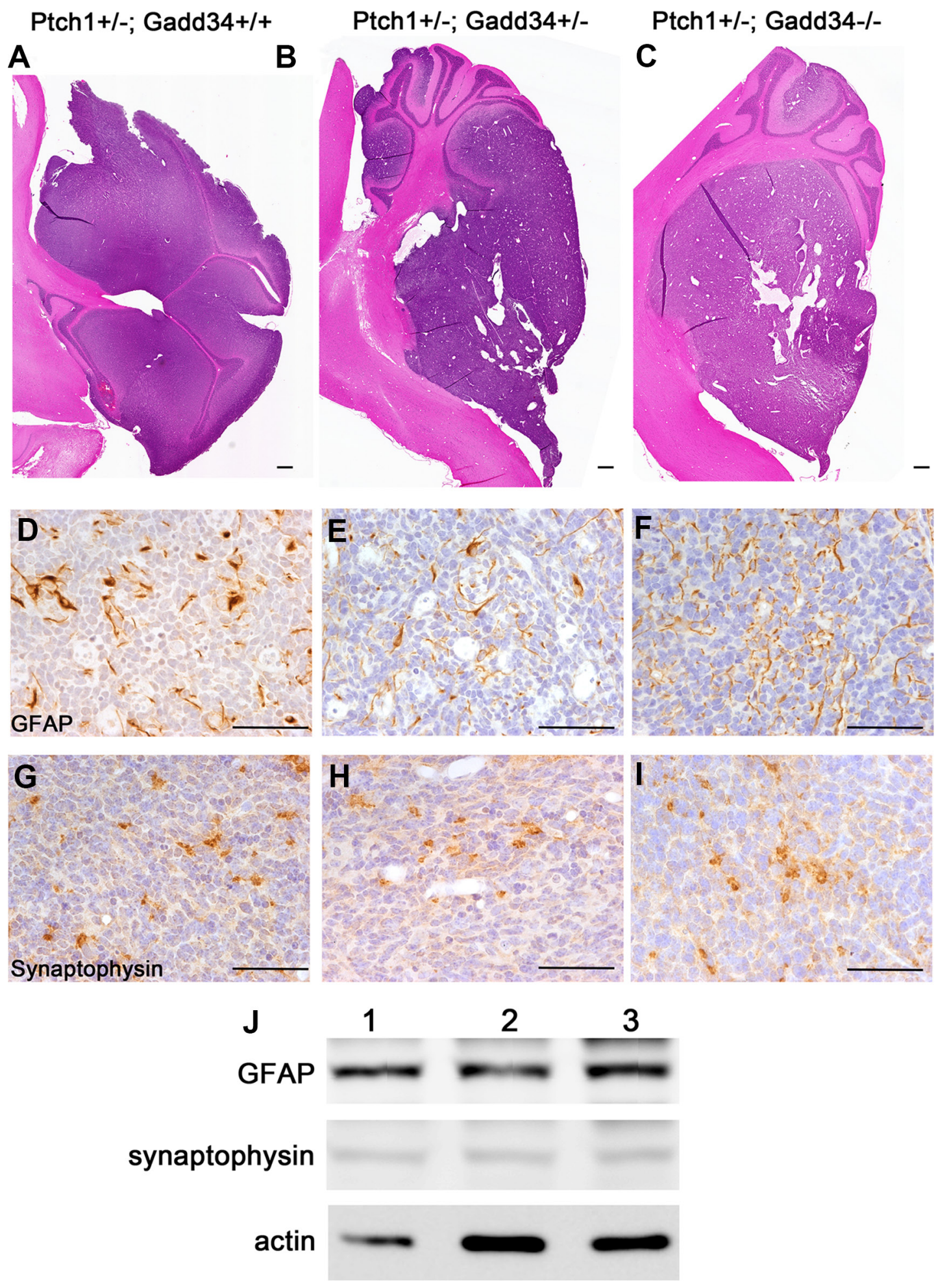

Figure 3: GADD34 mutation did not affect the morphology or differentiation of medulloblastoma in adult Ptch1+/mice. (A, B, C) H\&E staining showed that GADD34 mutation, either heterozygous or homozygous, did not change the morphology of medulloblastoma in adult Ptch1+/- mice. (D, E, F) GFAP IHC showed that GADD34 mutation, either heterozygous or homozygous, did not change the expression pattern of GFAP in medulloblastoma in adult Ptch1+/- mice. (G, H, I) Synaptophysin IHC showed that GADD34 mutation, either heterozygous or homozygous, did not change the expression pattern of synaptophysin in medulloblastoma in adult Ptch1+/- mice. (J) Western blot analysis showed that GADD34 mutation, either heterozygous or homozygous, did not change the protein level of GFAP or synaptophysin in the cerebellum of adult Ptch1+/- mice with medulloblastoma. 1, Ptch1+/-; Gadd34+/+ mice; 2, Ptch1+/-; Gadd34+/- mice; 3, Ptch1+/-; Gadd34-/- mice. $N=4$ animals. Scale bars: A-C, $1000 \mu \mathrm{m} ; \mathrm{D}-\mathrm{I}, 50 \mu \mathrm{m}$. 

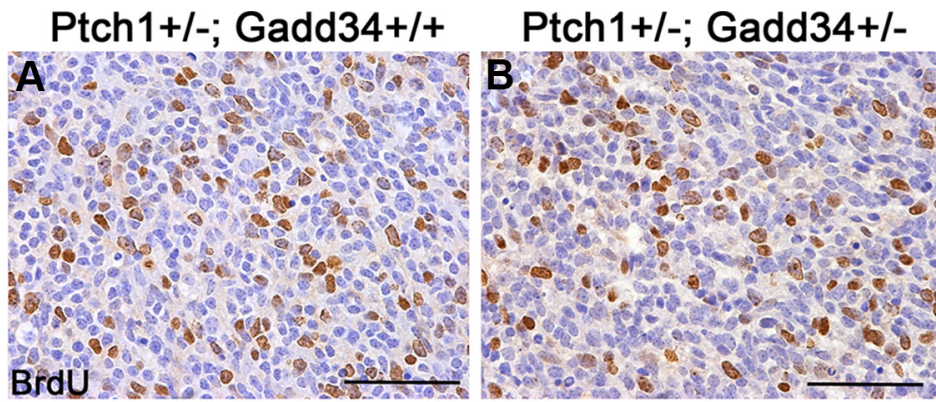

Ptch1+/-; Gadd34-/-
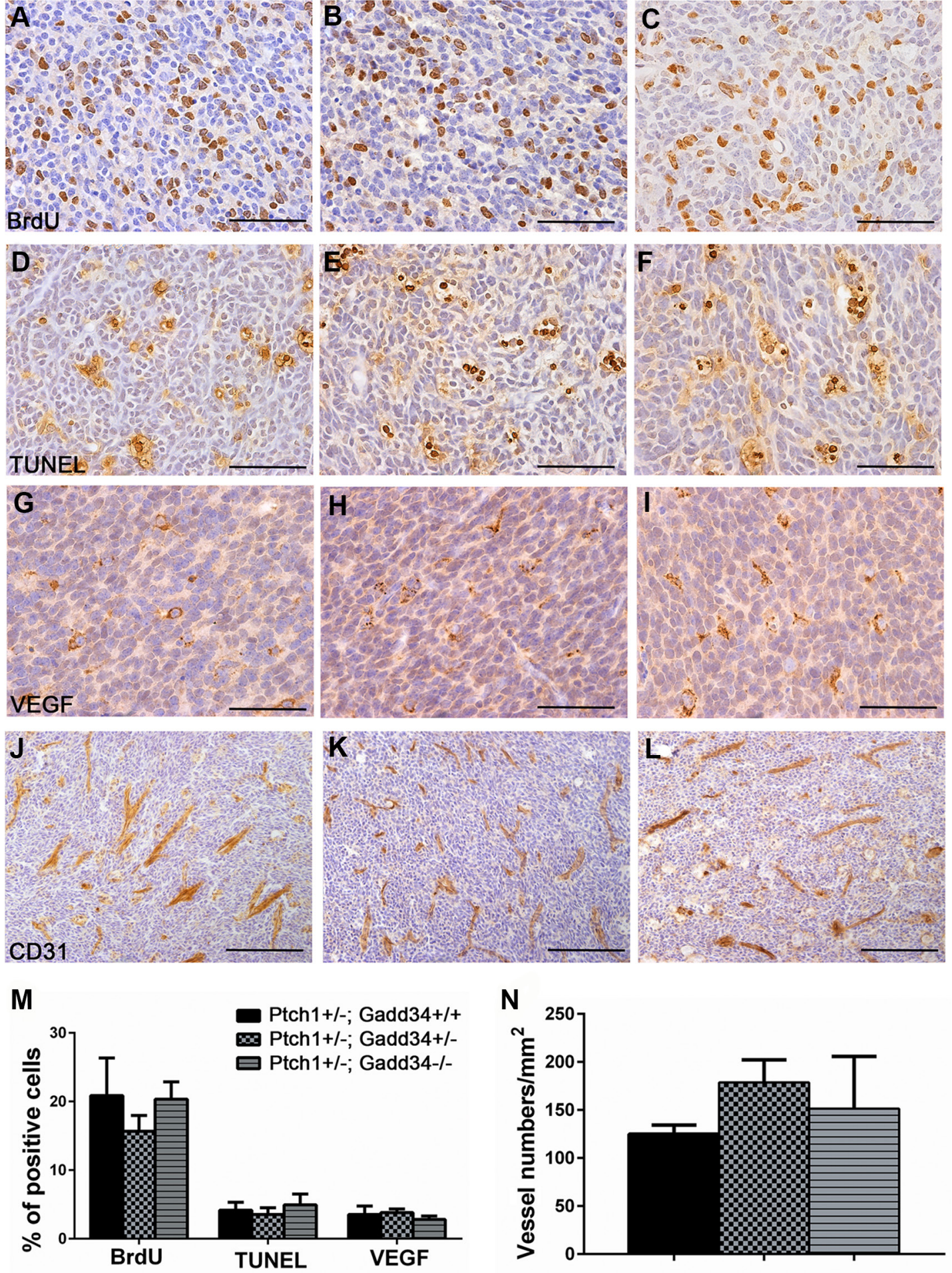

Figure 4: GADD34 mutation did not affect cell proliferation, cell apoptosis or angiogenesis in medulloblastoma in adult Ptch1+/- mice. (A, B, C, M) BrdU labeling revealed comparable number of proliferating cells in medulloblastoma in adult Ptch1+/-; Gadd34+/+ mice, Ptch1+/-; Gadd34+/- mice, and Ptch1+/-; Gadd34-/- mice. (D, E, F, M) TUNEL staining revealed comparable number of apoptotic cells in medulloblastoma in adult Ptch1+/-; Gadd34+/+ mice, Ptch1+/-; Gadd34+/- mice, and Ptch1+/-; Gadd34-/- mice. (G, H, I, M) VEGF-A IHC revealed comparable number of VEGF-A positive cells in medulloblastoma in adult Ptch1+/-; Gadd34+/+ mice, Ptch1+/-; Gadd34+/- mice, and Ptch1+/-; Gadd34-/- mice. (J, K, L, N) CD31 IHC revealed comparable number of blood vessels in medulloblastoma in adult Ptch1+/-; Gadd34+/+ mice, Ptch1+/-; Gadd34+/- mice, and Ptch1+/-; Gadd34-/- mice. $N=4$ animals. Error bars represent SD. Scale bars: (A-I), $50 \mu \mathrm{m} ;(\mathrm{J}-\mathrm{L}), 100 \mu \mathrm{m}$. 
lesions (Figure 5A-5C), which contained greater than 10 pre-malignant GCPs that were negative for NeuN (a marker of mature neurons) and positive for PCNA were counted as described in our previous paper [27]. We found that the number of hyperplastic lesions in the cerebellum of 6-week-old Ptch1+/-; Gadd34+/- mice was slightly increased as compared to Ptch1+/-; Gadd34+/+ mice (Figure 5D). Interestingly, the number of hyperplastic lesions in the cerebellum of 6-week-old Ptch1+/-; Gadd34-/- mice was significantly reduced as compared to Ptch1+/-; Gadd34+/+ mice (Figure 5D).

ATF4 IHC showed that immunoreactivity of ATF4 was noticeably increased in hyperplastic lesions in 6-week-old Ptch1+/-; Gadd34+/- mice as compared to Ptch1+/-; Gadd34+/+ mice (Figure 5E, 5F), and that the level of ATF4 was further increased in hyperplastic lesions in Ptch1+/-; Gadd34-/-mice (Figure 5G). BrdU labeling (Figure 6A-6C, 6G) showed that GADD34 mutation, either heterozygous or homozygous, did not significantly alter the rate of cell proliferation in hyperplastic lesions in 6-week-old Ptch1+/- mice. Importantly, TUNEL labeling showed that GADD34 homozygous mutation significantly increased the number of apoptotic cells in hyperplastic lesions in 6-week-old Ptch1+/- mice (Figure 6D, 6F, 6H). Nevertheless, GADD34 heterozygous mutation did not significantly change the number of apoptotic cells in hyperplastic lesions in 6-week-old Ptch $1+/-$ mice (Figure 6D, 6E, 6H). Taken together, our data suggest that the strongly enhanced ISR via GADD34 homozygous mutation significantly promotes pre-malignant GCP apoptosis and reduces the number of hyperplastic lesions in young Ptchl+/- mice, resulting in the significantly decreased incidence of medulloblastoma in adult Ptch $1+/-$ mice. Nevertheless, the moderately enhanced ISR via GADD34 heterozygous mutation slightly increases the number of hyperplastic lesions in young Ptch $1+/-$ mice and leads to the noticeably elevated incidence of medulloblastoma in adult Ptch $1+/-$ mice.

\section{DISCUSSION}

Evidence is emerging that the PERK-mediated ISR can either promote or suppress tumorigenesis by regulating cell apoptosis during the course of malignant transformation [12, 13, 14, 17]. Using PERK heterozygous deficient mice, our previous study showed that the decreased ISR reduces the incidence of medulloblastoma in Ptch $1+/-$ mice by enhancing pre-malignant GCP apoptosis during cell transformation [27]. In accordance with this previous study, we showed here that the moderately enhanced ISR via GADD34 heterozygous mutation slightly increased the number of hyperplastic lesions in young Ptch $1+/-$ mice and noticeably increased the incidence of medulloblastoma in Ptch1+/- mice. Nevertheless, we also found that the strongly enhanced ISR via GADD34 homozygous mutation significantly increased pre-malignant GCP apoptosis in hyperplastic lesions and reduced the lesion numbers in young Ptch $1+/-$ mice, resulting in the significant reduction of medulloblastoma incidence in adult Ptch $1+/-$ mice. Interestingly, neither GADD34 heterozygous mutation nor GADD34 homozygous mutation had an effect on medulloblastoma cells in adult Ptch $1+/-$ mice. Taken together, these findings suggest the dual role of the ISR in medulloblastoma tumorigenesis. Moderate activation of the ISR promotes medulloblastoma formation, but strong activation of the ISR suppresses the tumor formation.

Apoptosis functions as a natural barrier to malignant transformation [37, 38]. During the course of cell transformation, apoptosis triggered by cellular stresses counters unrestrained cell proliferation driven by mutation of oncogenes. ER stress is one of the cellular stresses that are activated in cells during malignant transformation. Recent studies showed that PERK activation in response to ER stress either promotes cell transformation by decreasing apoptosis or suppresses the transformation by increasing apoptosis [12, 13, 14, 17]. A number of studies showed that the effects of the PERKmediated ISR, beneficial or detrimental, on cells are activity-dependent $[18,39,40,41,42]$. The moderate ISR is beneficial to cells, but the strong ISR is detrimental to cells. Interestingly, our previous and current results indicate that the impact of the ISR on GCP apoptosis during cell transformation is also activity-dependent. Our previous paper showed that the decreased ISR via PERK heterozygous mutation enhances GCP apoptosis in hyperplastic lesions in young Ptch1+/- mice [27]. Herein, we showed that the strongly increased ISR via GADD34 homozygous mutation increased GCP apoptosis in hyperplastic lesions in young Ptch1+/- mice. Taken together, these data raise the possibility that the effects of the ISR, promoting or inhibiting, on tumorigenesis are determined by the activity of this pathway. Moderate activation of the ISR promotes cell transformation by suppressing apoptosis; however, strong activation of the ISR attenuates the transformation by facilitating apoptosis.

It was thought that strong activation of the ISR induces cell apoptosis through strong inhibition of protein biosynthesis and/or induction of CHOP [39, 43]. However, recent studies showed that CHOP induction is not necessary to lead to cell apoptosis $[42,44,45]$. We showed here that GADD34 homozygous mutation strongly increased the expression of CHOP in medulloblastoma but did not affect tumor cell apoptosis in adult Ptch $1+/-$ mice. Moreover, we found that GADD34 homozygous mutation strongly enhanced the expression of ATF4 and increased pre-malignant GCP apoptosis in hyperplastic lesions in young Ptch $1+/-$ mice. Nevertheless, IHC analysis showed that the immunoreactivity of CHOP was barely detectable in hyperplastic lesions in either young Ptch1+/-; Gadd34+/+ mice or Ptch1+/-; Gadd34-/- mice (data not shown). Clearly, there is no co-relationship between 
strong CHOP induction and cell apoptosis in either hyperplastic lesions in young Ptch1+/-; Gadd34-/- mice or medulloblastoma in adult mice. These data rule out the possibility that $\mathrm{CHOP}$ is a major player in regulating cell apoptosis during the development of medulloblastoma in Ptch1+/-; Gadd34-/- mice. Therefore, it is possible that the strongly increased ISR induces GCP apoptosis in hyperplastic lesions in young Ptch1+/-; Gadd34-/mice through strong inhibition of protein biosynthesis. Nevertheless, the molecular mechanisms by which the strongly increased ISR had no significant effect on medulloblastoma cells in adult Ptch1+/-; Gadd34-/mice are unknown and merit further investigation.

While we found that the incidence of medulloblastoma in Ptch1+/-; Gadd34+/- mice was noticeably increased as compared to Ptch1+/-; Gadd $34+/+$ mice $(42.6 \%$ vs $30.3 \%$, Figure $2 \mathrm{~B})$, the increase was not statistically significant. The conventional wisdom is that the value of the statistically non-significant data is in doubt. In this study, we used a large number of mice, around 70 mice per group. Moreover, the degree of increase (42.6\%, Ptch1+/-; Gadd $34+/-$ mice vs $30.3 \%$, Ptch1+/-; Gadd34+/+ mice) was quite impressive.
It is likely that the low incidence of medulloblastoma in Ptch $1+/-$ mice $(30.3 \%)$ is responsible for the statistically non-significant result. Therefore, we believed that it was necessary to include these important data in this report. Although the number of hyperplastic lesions in young Ptch1+/-; Gadd34+/- mice was slightly increased as compared to Ptch1+/-; Gadd34+/+ mice, we did not find evidence showing that GADD34 heterozygous mutation influenced pre-malignant GCP proliferation or apoptosis in the lesions. The mechanisms by which GADD34 heterozygous mutation noticeably enhances medulloblastoma tumorigenesis remain unknown. Clearly, the effects of GADD34 heterozygous mutation on medulloblastoma tumorigenesis warrant further investigation. A mouse model with a high incidence of medulloblastoma could be an ideal model to address this open question.

It has been shown that activation of the ISR in response to ER stress, which is induced by hypoxia and nutritional deficiency in the solid tumor microenvironment, plays a role in tumor development by regulating tumor cell viability, tumor invasion, and angiogenesis $[9,10,11]$. A report showed that GADD34 homozygous mutation
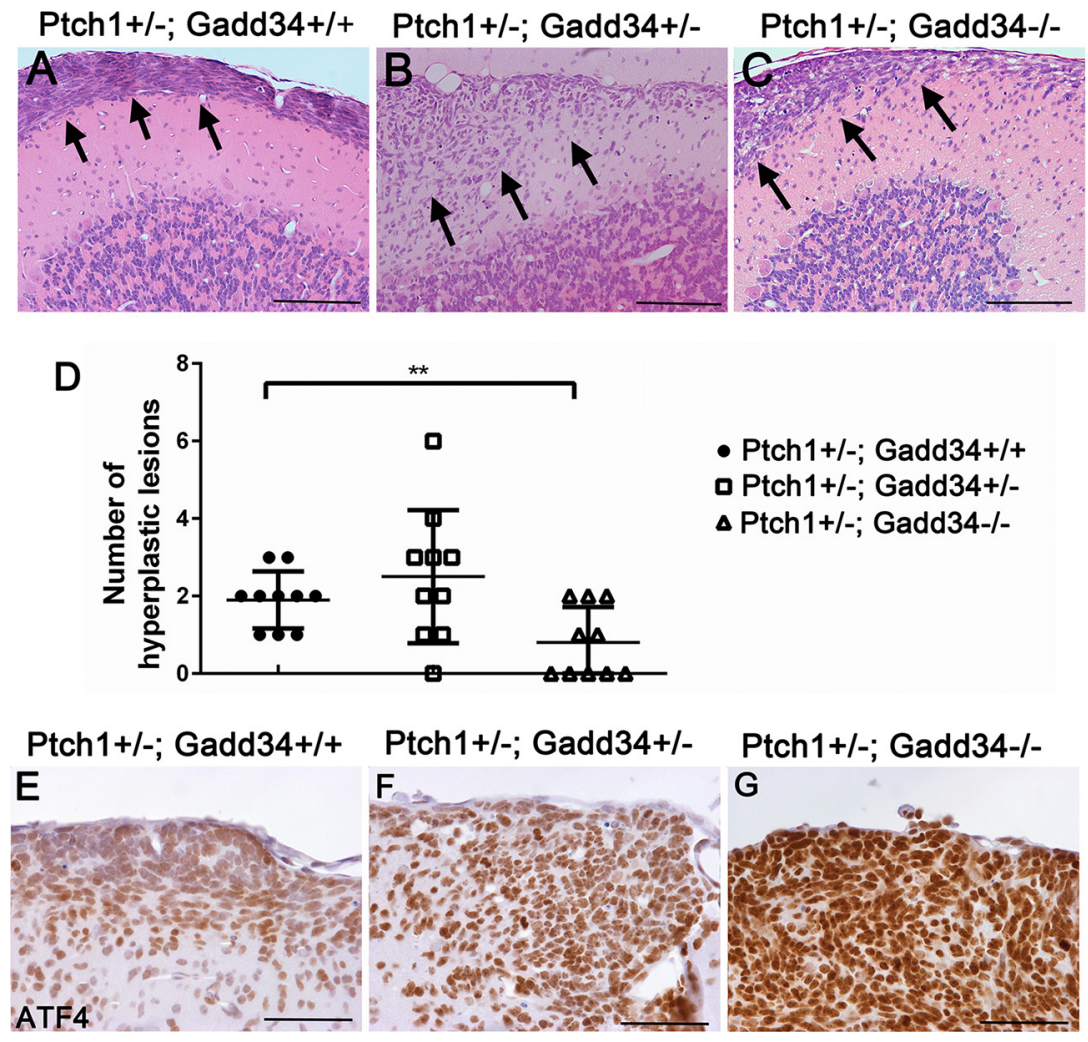

Figure 5: GADD34 mutation altered the number of cerebellar hyperplastic lesions in young Ptch1+/- mice. (A, B, C) H\&E staining revealed a hyperplastic lesion (arrows) in the cerebellum of 6-week-old Ptch1+/-; Gadd34+/+ mice, Ptch1+/-; Gadd34+/mice, and Ptch1+/-; Gadd34-/- mice. (D) The scatter plot showed that GADD34 heterozygous mutation slightly increased the number of hyperplastic lesions in the cerebellum of 6-week-old Ptch1+/- mice, and that GADD34 homozygous mutation significantly reduced the number of hyperplastic lesions in these mice. $(\mathbf{E}, \mathbf{F}, \mathbf{G})$ ATF4 IHC showed that the level of ATF4 was moderately increased in hyperplastic lesions in the cerebellum of 6-week-old Ptch1+/-; Gadd34+/- mice and strongly increased Ptch1+/-; Gadd34-/- mice, compared to Ptch1+/-; Gadd34+/+ mice. $N=10$ animals. Error bars represent SD, ${ }^{* *} P<0.01$. Scale bars: (A-C), $200 \mu \mathrm{m}$; (E-G) $50 \mu \mathrm{m}$. 
enhances the ISR, increases VEGF-A expression and angiogenesis, and facilitates medulloblastoma development in mice that ectopically express the immune cytokine interferon $-\gamma$ in the CNS during development [26]. Several studies also reported that the ISR stimulated the expression of VEGF-A in human medulloblastoma cells [46, 47]. Nevertheless, our previous study showed that PERK heterozygous deficiency does not influence cell proliferation or apoptosis, angiogenesis, or VEGF-A expression in medulloblastoma in adult Ptch $1+/-$ mice [27]. In accordance with this previous study, we showed herein that GADD34 mutation, either heterozygous or homozygous, did not alter cell proliferation, cell apoptosis, angiogenesis, or VEGF-A expression in medulloblastoma in adult Ptch1+/mice. Collectively, these data suggest that the effects of the
ISR on tumor cells are cell-context dependent, possibly determined by genetic mutations and/or epigenetic changes in individual molecular subtypes of tumors.

We showed that GADD34 mutation, either heterozygous or homozygous, elevated the level of CHOP but did not change the level of p-eIF2 $\alpha$ in medulloblastoma in adult Ptch $1+/-$ mice. The elevation of p-eIF $2 \alpha$ level is transient and highly dynamic in ERstressed cells. Moreover, there is a lot of feedback in the ISR. The steady state levels of any ISR markers reflect the composite effect of the primary defect (such as GADD34 mutation) and the feedback response, which may overshoot $[8,32]$. Therefore, it is not surprising that we found the lack of effect of the GADD34 mutation on steady state levels of p-eIF $2 \alpha$ in medulloblastoma in Ptch $1+/-$ mice.
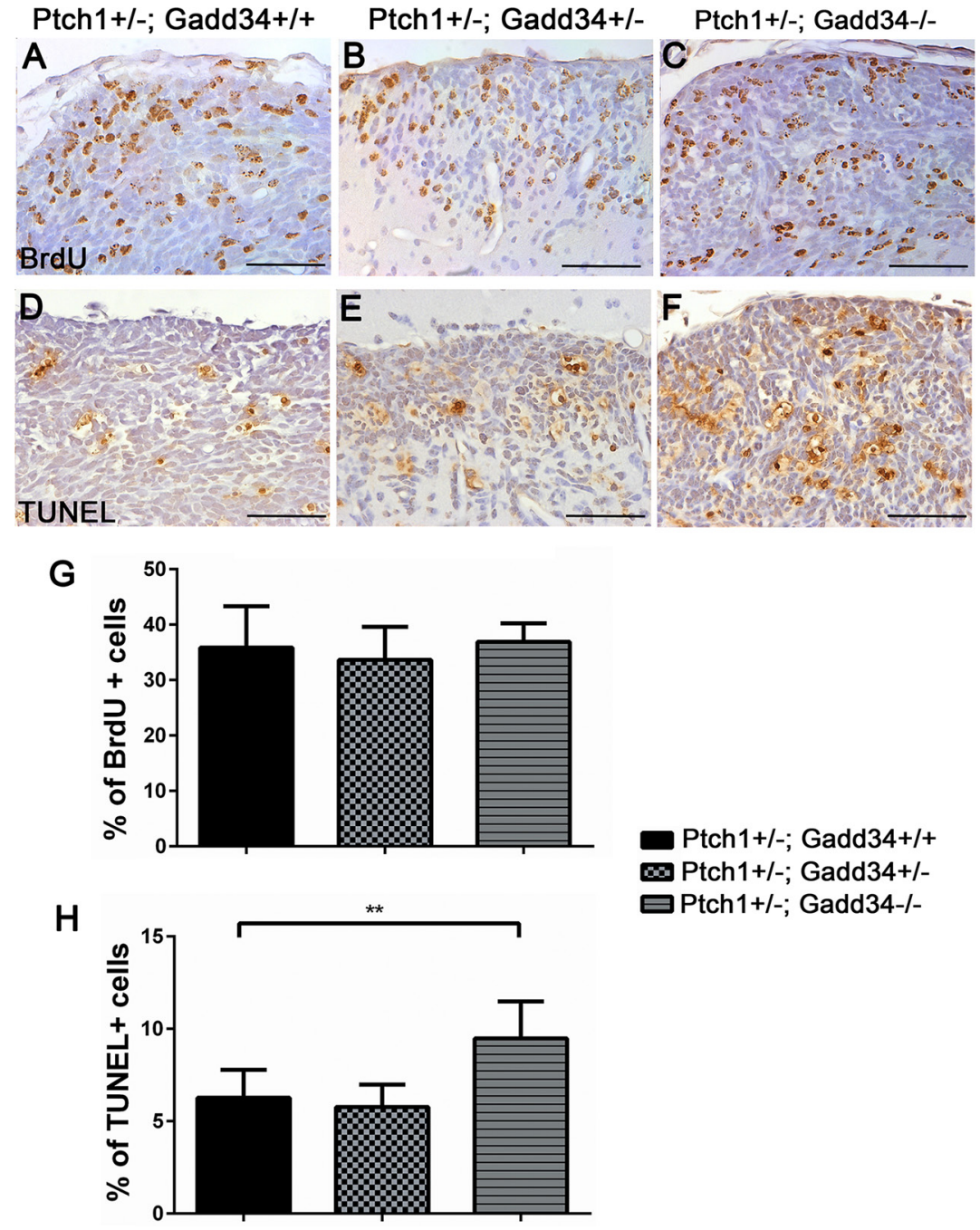

Ptch1+/-; Gadd34+/+

10 Ptch1+/-; Gadd34+/-

曰Ptch1+/-; Gadd34-/-

Figure 6: GADD34 homozygous mutation enhanced apoptosis of pre-malignant GCPs in hyperplastic lesions in the cerebellum of young Ptch1+/- mice. (A, B, C, G) BrdU labeling revealed comparable number of proliferating cells in hyperplastic lesions in the cerebellum of 6-week-old Ptch1+/-; Gadd34+/+ mice, Ptch1+/-; Gadd34+/- mice, and Ptch1+/-; Gadd34-/- mice. (D, E, F, H) TUNEL staining revealed significantly increased number of apoptotic cells in diffuse hyperplastic lesions in 6-week-old Ptch1+/-; Gadd34-/- mice compared to Ptch1+/-; Gadd34+/+ mice. However, GADD34 heterozygous mutation did not alter the number of apoptotic pre-malignant GCPs in hyperplastic lesions in Ptch $1+/-$ mice. $N=4$ animals. Error bars represent $\mathrm{SD},{ }^{* *} P<0.01$. Scale bars: (A-B), $50 \mu \mathrm{m}$. 
In summary, using GADD34 mutant mice, the results presented in this study advance our understanding of the effects on the PERK-mediated ISR on medulloblastoma development. Moderate increase of the ISR via GADD34 heterozygous mutation increased the incidence of medulloblastoma in Ptch1+/- mice. Nevertheless, strong increase of the ISR via GADD34 homozygous mutation decreased the incidence of medulloblastoma in Ptch1+/mice by enhancing pre-malignant GCPs apoptosis during the course of cell transformation. This work represents the first in vivo experimental demonstration of the dual role of the ISR on tumorigenesis.

\section{MATERIALS AND METHODS}

\section{Generation of mice}

Ptch $1+/-$ mice [21] on a mixed C57BL/6 x $129 \mathrm{~Sv}$ background were purchased from Jackson laboratory (Bar Harbor, Maine). Ptch1+/- mice were crossed with Gadd34-/- mice on the C57BL/6 background [7, 26], and then the resulting progeny were intercrossed to obtain Ptch1+/-; Gadd34+/+ mice, Ptch1+/-; Gadd34+/- mice as well as Ptch1+/-; Gadd34-/- mice. Genotypes were determined by PCR from DNA extracted from tail tips as described in previous papers [26, 27, 29]. Mice were monitored daily to detect medulloblastoma phenotypes, including ataxia, decreased movement, poor grooming, and doming of the skull, until the age of 8 months. All animal procedures were conducted in complete compliance with the NIH Guide for the Care and Use of Laboratory Animals and were approved by the Institutional Animal Care and Use Committee of the University of Minnesota.

\section{Histology, IHC, and TUNEL staining}

Adult mice with medulloblastoma phenotypes and all 8-month-old asymptomatic mice received an intraperitoneal injection of $100 \mathrm{mg} / \mathrm{kg}$ BrdU (SigmaAldrich, St. Louis, MO) $2 \mathrm{~h}$ prior to perfusion. Six-weekold asymptomatic mice received a BrdU injection $8 \mathrm{~h}$ prior to perfusion. Anesthetized mice were perfused through the left cardiac ventricle with $4 \%$ paraformaldehyde in $0.1 \mathrm{M}$ phosphate buffer. Brains were bisected in the sagittal plane, and one-half was postfixed for at least $48 \mathrm{~h}$ in $4 \%$ paraformaldehyde in PBS, dehydrated through graded alcohols, and embedded in paraffin. Serial sections of $5 \mu \mathrm{m}$ thickness were cut and every tenth section in the series was routinely stained with $\mathrm{H} \& \mathrm{E}$. The other half of the brain was postfixed for $1 \mathrm{~h}$ in $4 \%$ paraformaldehyde in PBS, cryopreserved in 30\% sucrose for $48 \mathrm{~h}$, embedded in OCT compound, and frozen on dry ice. Frozen sections were cut in a cryostat at $10 \mu \mathrm{m}$ thickness. IHC for NeuN (1: 200, Millipore, Temecula, CA), PCNA (1:50,000, Sigma-Aldrich), GFAP (1:1000, Covance, Princeton, NJ), synaptophysin (1:200, Millipore), VEGF-A (1:50,
Santa Cruz Biotechnology, Dallas, TX), and ATF4 (1:100, Abcam, Cambridge, MA) were performed on paraffin sections. IHC for CD31 (1:50, Santa Cruz Biotechnology) was performed on cryosections. All primary antibodies were detected using the Vectastain ABC kits (Vector Laboratories, Burlingame, CA) and 3'5'-diaminobenzidine/ $\mathrm{H}_{2} \mathrm{O}_{2}$ reagent (Vector Laboratories) as substrate.

TUNEL staining was performed on paraffin sections using the ApopTag kit (Millipore) according to the manufacturer's instructions. IHC for BrdU (1:1000; Sigma-Aldrich), was performed on paraffin sections as described in our previous papers. We quantified positive cells for BrdU, TUNEL and VEGF-A as well as CD31 positive blood vessels in the center of medulloblastoma or hyperplastic lesions in the cerebellum as described in our previous papers $[26,27,48]$.

\section{Western blot analysis}

Cerebellar tissues were removed from adult control wild type mice as well as adult symptomatic Ptch1+/-; Gadd34+/+ mice, Ptch1+/-; Gadd34+/mice, and Ptch1+/-; Gadd34 -/- mice, which displayed typical medulloblastoma clinical phenotypes and enlarged cerebellum. These tissues were homogenized in 5 volumes of Triton X-100 buffer using a motorized homogenizer as previously described [27, 49]. After incubation on ice for $15 \mathrm{~min}$, the extracts were cleared by centrifugation at $14,000 \mathrm{rpm}$ for $30 \mathrm{~min}$ twice. The protein concentration of each extract was determined by DC Protein Assay (Bio-Rad Laboratories, Hercules, CA). The extracts $(120 \mu \mathrm{g})$ were separated by SDS-PAGE and transferred to nitrocellulose membranes. The blots were then incubated with primary antibodies to p-eIF $2 \alpha$ (1:1000, Cell Signaling Technology, Danvers, MA), eIF2 $\alpha$ (1:1000, Santa Cruz Biotechnology), ATF4 (1:1000, Abcam), CHOP (1:1000, Thermo Scientific, Grand Island, NY), GFAP (1:1000, Covance), synaptophysin (1:1000, Millipore), and actin (1:5000, Sigma-Aldrich), followed by the HRP-conjugated secondary antibody (Vector Laboratories). The chemiluminescent signal was detected by the ECL Detection Reagents (GE Healthcare Biosciences, Piscataway, NJ). The intensity of the recorded chemiluminescence signal was quantified using the ImageQuantTL software from GE Healthcare Life Sciences.

\section{Statistical methods}

Data were expressed as mean \pm standard deviation (SD). Comparisons between multiple groups were statistically evaluated by the one-way ANOVA test using Prism 6 (GraphPad, Software Inc., La Jolla, CA). The incidence of symptomatic medulloblastoma between Ptch1+/-; Gadd34+/+ mice and Ptch1+/-; Gadd34+/mice as well as between Ptch1+/-; Gadd34+/+ mice and 
Ptch1+/-; Gadd34-/- mice was statistically evaluated by Kaplan-Meier analysis and the incidence of total medulloblastoma between two groups was statistically evaluated by $\chi^{2}$ test using Prism 6 (GraphPad). $P<0.05$ was considered significant.

\section{CONFLICTS OF INTEREST}

The authors declare no competing financial interests.

\section{GRANT SUPPORT}

Wensheng Lin is supported by grants from the National Institutes of Health (NS073132 and NS094151) and the National Multiple Sclerosis Society (RG4813-A-2 and RG5239-A-3). David Ron is a Principal Research Fellow of the Wellcome Trust (GR-084812 and GR-100140).

\section{REFERENCES}

1. Harding HP, Zhang Y, Zeng H, Novoa I, Lu PD, Calfon M, Sadri N, Yun C, Popko B, Paules R, Stojdl DF, Bell JC, Hettmann T, et al. An integrated stress response regulates amino acid metabolism and resistance to oxidative stress. Mol Cell. 2003;11:619-33.

2. Lu PD, Harding HP, Ron D. Translation reinitiation at alternative open reading frames regulates gene expression in an integrated stress response. J Cell Biol. 2004; 167:27-33.

3. Proud CG. eIF2 and the control of cell physiology. Semin Cell Dev Biol. 2005; 16:3-12.

4. Connor JH, Weiser DC, Li S, Hallenbeck JM, Shenolikar S. Growth arrest and DNA damage-inducible protein GADD34 assembles a novel signaling complex containing protein phosphatase 1 and inhibitor 1. Mol Cell Biol. 2001; 21:6841-50.

5. Novoa I, Zeng H, Harding HP, Ron D. Feedback inhibition of the unfolded protein response by GADD34-mediated dephosphorylation of eIF2alpha. J Cell Biol. 2001; 153:1011-22.

6. Novoa I, Zhang Y, Zeng H, Jungreis R, Harding HP, Ron D. Stress-induced gene expression requires programmed recovery from translational repression. EMBO J. 2003; 22:1180-7.

7. Marciniak SJ, Yun CY, Oyadomari S, Novoa I, Zhang Y, Jungreis R, Nagata K, Harding HP, Ron D. CHOP induces death by promoting protein synthesis and oxidation in the stressed endoplasmic reticulum. Genes Dev. 2004;18:3066-77.

8. Walter P, Ron D. The unfolded protein response: from stress pathway to homeostatic regulation. Science. 2011; 334:1081-6.

9. Wang M, Kaufman RJ. The impact of the endoplasmic reticulum protein-folding environment on cancer development. Nat Rev Cancer. 2014; 14:581-97.
10. Bi M, Naczki C, Koritzinsky M, Fels D, Blais J, Hu N, Harding H, Novoa I, Varia M, Raleigh J, Scheuner D, Kaufman RJ, Bell J, et al. ER stress-regulated translation increases tolerance to extreme hypoxia and promotes tumor growth. EMBO J 2005; 24:3470-81.

11. Moenner M, Pluquet O, Bouchecareilh M, Chevet E. Integrated endoplasmic reticulum stress responses in cancer. Cancer Res. 2007; 67:10631-634.

12. Hart LS, Cunningham JT, Datta T, Dey S, Tameire F, Lehman SL, Qiu B, Zhang H, Cerniglia G, Bi M, Li Y, Gao Y, Liu H, et al. ER stress-mediated autophagy promotes Myc-dependent transformation and tumor growth. J Clin Invest. 2012; 122:4621-34.

13. Huber AL, Lebeau J, Guillaumot P, Pétrilli V, Malek M, Chilloux J, Fauvet F, Payen L, Kfoury A, Renno T, Chevet E, Manié SN. p58(IPK)-mediated attenuation of the proapoptotic PERK-CHOP pathway allows malignant progression upon low glucose. Mol Cell. 2013; 49:1049-59.

14. Feng YX, Sokol ES, Del Vecchio CA, Sanduja S, Claessen JH, Proia TA, Jin DX, Reinhardt F, Ploegh HL, Wang Q, Gupta PB. Epithelial-to-mesenchymal transition activates PERK-eIF2 $\alpha$ and sensitizes cells to endoplasmic reticulum stress. Cancer Discov. 2014; 4:702-15.

15. Ma Y, Hendershot LM. The role of the unfolded protein response in tumour development: friend or foe? Nat Rev Cancer. 2004; 4:966-77.

16. Vandewynckel YP, Laukens D, Geerts A, Bogaerts E, Paridaens A, Verhelst X, Janssens S, Heindryckx F, Van Vlierberghe $H$. The paradox of the unfolded protein response in cancer. Anticancer Res. 2013; 33:4683-94.

17. Chevet E, Hetz C, Samali A. Endoplasmic reticulum stressactivated cell reprogramming in oncogenesis. Cancer Discov. 2015; 5:586-97.

18. Ranganathan AC, Ojha S, Kourtidis A, Conklin DS, Aguirre-Ghiso JA. Dual function of pancreatic endoplasmic reticulum kinase in tumor cell growth arrest and survival. Cancer Res. 2008; 68:3260-68.

19. Northcott PA, Jones DT, Kool M, Robinson GW, Gilbertson RJ, Cho YJ, Pomeroy SL, Korshunov A, Lichter P, Taylor MD, Pfister SM. Medulloblastomics: the end of the beginning. Nat Rev Cancer. 2012; 12:818-34.

20. Taylor MD, Northcott PA, Korshunov A, Remke M, Cho YJ, Clifford SC, Eberhart CG, Parsons DW, Rutkowski S, Gajjar A, Ellison DW, Lichter P, Gilbertson RJ, et al. Molecular subgroups of medulloblastoma: the current consensus. Acta Neuropathol. 2012; 123:465-72.

21. Goodrich LV, Milenković L, Higgins KM, Scott MP. Altered neural cell fates and medulloblastoma in mouse patched mutants. Science. 1997; 277:1109-13

22. Corcoran RB, Scott MP. A mouse model for medulloblastoma and basal cell nevus syndrome. J Neurooncol. 2001; 53:307-18

23. Oliver TG, Read TA, Kessler JD, Mehmeti A, Wells JF, Huynh TT, Lin SM, Wechsler-Reya RJ. Loss of patched and 
disruption of granule cell development in a pre-neoplastic stage of medulloblastoma. Development. 2005; 132:2425-39.

24. Corcoran RB, Bachar Raveh T, Barakat MT, Lee EY, Scott MP. Insulin-like growth factor 2 is required for progression to advanced medulloblastoma in patched1 heterozygous mice. Cancer Res. 2008; 68:8788-95.

25. Farioli-Vecchioli S, Cinà I, Ceccarelli M, Micheli L, Leonardi L, Ciotti MT, De Bardi M, Di Rocco C, Pallini R, Cavallaro S, Tirone F. Tis21 knock-out enhances the frequency of medulloblastoma in Patched1 heterozygous mice by inhibiting the Cxcl3-dependent migration of cerebellar neurons. J Neurosci. 2012; 32:15547-64.

26. Lin W, Lin Y, Li J, Harding HP, Ron D, Jamison S. A deregulated integrated stress response promotes interferon$\gamma$-induced medulloblastoma. J Neurosci Res. 2011; 89:1586-95.

27. Ho Y, Li X, Jamison S, Harding HP, McKinnon PJ, Ron D, Lin W. PERK Activation Promotes Medulloblastoma Tumorigenesis by Attenuating Premalignant Granule Cell Precursor Apoptosis. Am J Pathol. 2016; 186:1939-51.

28. Fults DW: Modeling medulloblastoma with genetically engineered mice. Neurosurg Focus. 2005; 19:E7.

29. Lin W, Kunkler PE, Harding HP, Ron D, Kraig RP, Popko B. Enhanced integrated stress response promotes myelinating oligodendrocyte survival in response to interferon-gamma. Am J Pathol. 2008; 173:1508-17.

30. Way SW, Popko B. Harnessing the integrated stress response for the treatment of multiple sclerosis. Lancet Neurol. 2016; 15:434-43.

31. Wang L, Popko B, Roos RP. An enhanced integrated stress response ameliorates mutant SOD1-induced ALS. Hum Mol Genet. 2014; 23:2629-38.

32. Pavitt GD, Ron D. New insights into translational regulation in the endoplasmic reticulum unfolded protein response. Cold Spring Harb Perspect Biol. 2012; 4. pii:a012278.

33. Atkins C, Liu Q, Minthorn E, Zhang SY, Figueroa DJ, Moss K, Stanley TB, Sanders B, Goetz A, Gaul N, Choudhry AE, Alsaid H, Jucker BM, et al. Characterization of a novel PERK kinase inhibitor with antitumor and antiangiogenic activity. Cancer Res. 2013; 73:1993-2002.

34. Wechsler-Reya R, Scott MP. The developmental biology of brain tumors. Annu Rev Neurosci. 2001; 24:385-428.

35. Kim JY, Nelson AL, Algon SA, Graves O, Sturla LM, Goumnerova LC, Rowitch DH, Segal RA, Pomeroy SL. Medulloblastoma tumorigenesis diverges from cerebellar granule cell differentiation in patched heterozygous mice. Dev Biol. 2003; 263:50-66.

36. Mille F, Tamayo-Orrego L, Lévesque M, Remke M, Korshunov A, Cardin J, Bouchard N, Izzi L, Kool M,
Northcott PA, Taylor MD, Pfister SM, Charron F. The Shh receptor Boc promotes progression of early medulloblastoma to advanced tumors. Dev Cell. 2014; 31:34-47.

37. Hanahan D, Weinberg RA. The hallmarks of cancer. Cell. 2000; 100:57-70.

38. Hanahan D, Weinberg RA. Hallmarks of cancer: the next generation. Cell. 2011; 144:646-74.

39. Tabas I, Ron D. Integrating the mechanisms of apoptosis induced by endoplasmic reticulum stress. Nat Cell Biol. 2011; 13:184-90.

40. Lin W, Lin Y, Li J, Fenstermaker AG, Way SW, Clayton B, Jamison S, Harding HP, Ron D, Popko B. Oligodendrocytespecific activation of PERK signaling protects mice against experimental autoimmune encephalomyelitis. J Neurosci. 2013; 33:5980-91.

41. Lin Y, Huang G, Jamison S, Li J, Harding HP, Ron D, Lin W. PERK activation preserves the viability and function of remyelinating oligodendrocytes in immune-mediated demyelinating diseases. Am J Pathol. 2014; 184:507-19.

42. Lin Y, Pang X, Huang G, Jamison S, Fang J, Harding HP, Ron D, Lin W. Impaired eukaryotic translation initiation factor 2B activity specifically in oligodendrocytes reproduces the pathology of vanishing white matter disease in mice. J Neurosci. 2014; 34:12182-91.

43. Hetz C. The unfolded protein response: controlling cell fate decisions under ER stress and beyond. Nat Rev Mol Cell Biol. 2012; 13:89-102.

44. Gow A, Wrabetz L. CHOP and the endoplasmic reticulum stress response in myelinating glia. Curr Opin Neurobiol. 2009; 19:505-10.

45. Stone $\mathrm{S}, \mathrm{Lin} \mathrm{W}$. The unfolded protein response in multiple sclerosis. Front Neurosci. 2015; 9:264.

46. Jamison S, Lin Y, Lin W. Pancreatic endoplasmic reticulum kinase activation promotes medulloblastoma cell migration and invasion through induction of vascular endothelial growth factor A. PLoS One. 2015; 10:e0120252.

47. Pereira ER, Liao N, Neale GA, Hendershot LM. Transcriptional and post-transcriptional regulation of proangiogenic factors by the unfolded protein response. PLoS One. 2010; 5:e12521.

48. Lin W, Kemper A, McCarthy KD, Pytel P, Wang JP, Campbell IL, Utset MF, Popko B. Interferon-gamma induced medulloblastoma in the developing cerebellum. J Neurosci. 2004; 24:10074-83.

49. Lin $\mathrm{Y}$, Jamison $\mathrm{S}$, Lin W. Interferon- $\gamma$ activates nuclear factor- $\kappa \mathrm{B}$ in oligodendrocytes through a process mediated by the unfolded protein response. PLoS One. 2012; 7:e36408. 